\title{
Género y apropiación de la perspectiva de inversión social en Bolivia: el sistema de los Bonos
}

Genre et appropriation de la perspective d'investissement social en Bolivie : le système des bons

Gender and appropriation of a social investment perspective in Bolivia: the system of Bonos

\section{Nora Nagels}

\section{OpenEdition}

\section{Journals}

Edición electrónica

URL: http://journals.openedition.org/bifea/7651

DOI: $10.4000 /$ bifea.7651

ISSN: 2076-5827

\section{Editor}

Institut Français d'Études Andines

\section{Edición impresa}

Fecha de publicación: 1 diciembre 2015

Paginación: 365-385

ISSN: 0303-7495

\section{Referencia electrónica}

Nora Nagels, «Género y apropiación de la perspectiva de inversión social en Bolivia: el sistema de los Bonos », Bulletin de l'Institut français d'études andines [En línea], 44 (3) | 2015, Publicado el 08 diciembre 2015, consultado el 06 noviembre 2020. URL : http://journals.openedition.org/bifea/7651 ; DOI : https://doi.org/10.4000/bifea.7651

\section{(c) $(1) \odot$}

Les contenus du Bulletin de l'Institut français d'études andines sont mis à disposition selon les termes de la licence Creative Commons Attribution - Pas d'Utilisation Commerciale - Pas de Modification 4.0 International. 


\section{Género y apropiación de la perspectiva de inversión social en Bolivia: el sistema de los Bonos}

\section{Nora Nagels}

\section{Resumen}

Como la mayoría de los países latinoamericanos, Bolivia adoptó un nuevo instrumento de asistencia social considerado en la escena internacional del desarrollo como la mejor manera de luchar contra la pobreza: los programas de transferencias condicionadas monetarias (TCM ). Pertenecen a la perspectiva de inversión social Posconsenso de Washington. De acuerdo con esta perspectiva, los TCM tienen como objetivo a corto plazo reducir la pobreza por el aumento de la liquidez monetaria en los hogares y a largo plazo, romper con el ciclo intergeneracional de la pobreza mediante la inversión en el capital humano de las generaciones futuras. La apropiación de esta perspectiva en Bolivia por el sistema de los Bonos se construye mediante el Estado Plurinacional que valora los derechos colectivos indígenas. A partir de una metodología cualitativa de análisis del discurso, esta contribución estudia la configuración «desarrollista» de los Bonos y las interacciones entre sus responsables políticos y destinatarias. El análisis de las entrevistas con los responsables políticos y las mujeres receptoras muestra que en Bolivia este tipo de intervención reproduce las antiguas características de las políticas sociales latinoamericanas: el maternalismo y el neocolonialismo.

Palabras clave: género, programa de transferencias monetarias condicionadas (TMC), inversión social, políticas sociales, neocolonialismo

* Profesora, Departamento de Ciencias Políticas de la Universidad del Q uébec en Montréal. E-mail: Nagels.nora@uqam.ca 


\title{
Genre et appropriation de la perspective d'investissement social en Bolivie : le système des bons
}

\author{
Résumé
}

Comme la majorité des pays d'Amérique latine, la Bolivie a adopté un nouvel instrument de politiques d'assistance sociale considéré comme le meilleur moyen de lutte contre la pauvreté sur la scène internationale du développement: les programmes de transferts conditionnés (PTC). Ces derniers appartiennent à la perspective d'investissement social post-Consensus de Washington. En accord avec cette perspective, les PTC visent, à court terme, à réduire la pauvreté par l'augmentation des liquidités monétaires dans les ménages et, à long terme, rompre le cycle intergénérationnel de la pauvreté par l'investissement dans le capital humain des générations futures. L'appropriation de cette perspective en Bolivie par le système des bons s'articule avec le nouvel État plurinational qui valorise les droits collectifs autochtones. À partir d'une méthodologie qualitative d'analyse de discours, cette contribution étudie la configuration « développementaliste » des bons et les interactions entre ses responsables politiques et ses récipiendaires. L'analyse des entretiens avec les décideurs politiques et les femmes récipiendaires montre qu'en Bolivie ce type d'intervention reproduit les caractéristiques anciennes des politiques sociales latino-américaines : le maternalisme et le néocolonialisme.

Mots-clés : genre, programme de transferts monétaires condititionnés (TMC), investissement social, politiques sociales, néocolonialisme

\section{Gender and appropriation of a social investment perspective in Bolivia: the system of Bonos}

\begin{abstract}
Like most Latin American countries, Bolivia adopted a new instrument of social assistance policies that was considered as the best way to fight poverty in the international area of development: Conditional Cash Transfer programs (CCT). These belong to the social investment perspective in the post-Washington Consensus. According to this perspective, CCT's aim in the short term was to reduce poverty by increasing the money supply of households and in the long-term, it was to break the intergenerational cycle of poverty by investing in human capital for future generations. The appropriation of this perspective in Bolivia with the Bonos' system was mediated by the new Plurinational State that included indigenous collective rights. Using a qualitative methodology of discourse analysis, this paper studies the "developmentalist" configuration of the Bonos and the interactions among policymakers and women recipients. An analysis of the interviews with policy makers and women recipients highlights that in Bolivia this new perspective reproduced old features of Latin American social policies: maternalism and neocolonialism.
\end{abstract}

Keywords: gender, conditional Cash Transfer (CCT), social investment, social policies, neocolonialism

\section{INTRODUCCIÓN}

Los programas de transferencias monetarias condicionadas (TCM ) se han extendido en América Latina como respuesta a la constatación de una falta generalizada de crecimiento económico y reducción de la pobreza de parte del modelo neoliberal. 
En la región, los responsables políticos buscaron un enfoque de política social más global que los prevalentes durante el Consenso de Washington. Los TM C Ilenaron el vacío identificado. Son considerados como el mayor cambio en las políticas sociales de América Latina en los últimos veinte años (Barrientos, 2009; Martinez, 2008) y pertenecen a la perspectiva de inversión social (Jenson, 2010; Staab, 2010; Razavi, 2014; Lopreite \& Macdonald, 2014). La perspectiva de inversión social da forma a la estructura cognitiva de las políticas sociales en el Sur y el Norte promoviendo la inversión en el capital humano de las generaciones futuras (Jenson \& Saint-M artin, 2006).

Lasintervenciones en salud y educación de losTCM se presentan como apoyos para la adquisición de capital humano, con los efectos previstos de reducir la pobreza y romper el ciclo intergeneracional de la pobreza. En la escena internacional de desarrollo, son considerados como la «mejor práctica» para reducir la pobreza. Tanto las organizaciones financieras internacionales como el Banco M undial, el Fondo M onetario Internacional que las agencias de la $\mathrm{O}$ rganización de las $\mathrm{N}$ aciones Unidas - como el PNUD, la OIT y la CEPAL- u organismos independientes como el IFRI 0 el Wilson Center o también académicos estiman que los PTC son eficientes en términos de sus objetivos en educación (O IT \& Bachelet, 2011; Barrientos \& Dejong, 2006; Fiszbein et al., 2009); salud (Villatoro, 2004; Skoufias \& Clafferty, 2001), pobreza y desigualdades (The economist, 2010; Cecchini \& Madariaga, 2011; Bastagli, 2009).

Entonces, los actores nacionales identifican los TCM como la «nueva norma profesional en la comunidad del desarrollo» (Sugiyama, 2011: 262) y los adoptan. Sin embargo, lejos de ser impuestos desde las organizaciones internacionales de desarrollo y ser adoptados tal cual al nivel nacional, los TCM son traducidos no solo en los regímenes políticos ( $\mathrm{N}$ agels, 2014) pero también en su implementación local. A partir del análisis de la implementación de los TCM bolivianos - los Bonos- este artículo mitiga el consenso positivo que considera los TCM como mejores prácticas de lucha contra la pobreza. El argumento principal es que la traducción local de este nuevo instrumento de políticas sociales reproduce características antiguas de políticas sociales latinoamericanistas: el maternalismo y el neocolonialismo. Los Bonos reproducen las desigualdades de género y son utilizados como instrumentos coercitivos con fines higienistas. Las recipientes indígenas no son víctimas meramente pasivas de estas relaciones jerárquicas sociales, sino que participan a su dominación y también construyen espacios de resistencia.

Este argumento se basa en un enfoque socio-antropológico del desarrollo. Este enfoque no es normativo pero empírico y fundamental. El desarrollo es un objeto (y no una teoría) de la antropología académica. El objetivo no es salvar o condenar el desarrollo sino comprender prácticas sociales complejas.

El desarrollo es de hecho no otra cosa que el conjunto de actuaciones de todo tipo que se dicen ser cerca o lejos de él (desde el «desarrollador», así como del «desarrollado») en la diversidad de sus supuestos, significados y prácticas. La existencia de una «configuración desarrollista» - el complejo conjunto de instituciones, flujos y actores para quienes el desarrollo es 
un recurso, un trabajo, un mercado, un problema, o una estrategia- es suficiente para justificar la existencia de una socio-antropología que toma el desarrollo como un objeto de estudio (Olivier de Sardan, 2001: 731).

Este enfoque analiza las interacciones entre «desarrolladores» y «desarrollados». Está basado en el interaccionismo metodológico donde las interacciones sociales se consideran como «entradas empíricas privilegiadas mientras se niega a que sean un objeto en sí mismo, es decir, sin limitarse a ellas o atrapado por ellas» (O liver de Sardan, 2001: 742).

Las políticas de desarrollo son las solapadas de las políticas públicas locales y separar la configuración «desarrollista» y las acciones estatales «día a día» es complicado (O liver de Sardan, 2001: 746). La socio-antropología del desarrollo es cada vez más vinculada a una socio-antropología de los cambios sociales. Entonces, los TCM, como políticas públicas influenciadas por la esfera internacional del desarrollo, pueden ser analizados con este enfoque. «Cerca del campo», la socio-antropología se basa en un método cualitativo con el objetivo de comprender el sentido dado por los actores a sus acciones. Aquí la configuración desarrollista de los TCM bolivianos es analizada a través de un análisis cualitativo del discurso. Entre 2008 y 2010, alrededor de 50 entrevistas se llevaron a cabo con los responsables políticos y las receptoras de los Bonos en La Paz y El Alto1. El estudio de las interacciones entre «desarrolladores» y «desarrollados» se basa en el análisis de las percepciones y representaciones de los responsables políticos y los receptores sobre los demás. Las citaciones movilizadas ilustran discursos masivos que no son marginales. El uso del análisis del discurso en estas entrevistas proporciona acceso a las representaciones sociales (Jodelet, 1989) de género y de las relaciones étnicas construidas por los actores claves, los Bonos.

Este trabajo muestra resultados imprevistos en la población focalizada y brechas entre las normas de los TCM, perteneciendo a la perspectiva de inversión social, y las normas locales. Las recipientes de los Bonos oscilan entre la reproducción y la resistencia a estas normas que las dominan como mujeres indígenas. Este artículo se divide en tres partes. En primer lugar, se describe el contexto boliviano de adopción de los TMC. En segundo lugar, se analiza el consenso acerca de las representaciones de género. En tercer lugar, se consideran las brechas, tensiones, resistencias y los resultados no previstos de los Bonos en su implementación local. En la tercera parte, se discute cómo estos programas refuerzan dominaciones sociales, étnicas y de género.

1 Esta investigación es parte de una tesis doctoral titulada «Género y políticas de lucha contra la pobreza en Perú y Bolivia: desafíos de ciudadana» a donde se explicó la posición situada de la autora en la co-construcción de conocimientos (Nagels, 2013). El objetivo de este trabajo es confrontar las representaciones de la pobreza, de las mujeres pobres y de las relaciones Estado-sociedad construidas por los actores - desde los políticos hacia las recipientes- de diferentes programas sociales en los dos países. El trabajo de campo se hizo en La Paz, El Alto, Lima y Ayacucho. Se basó en el método de la «bola de nieve»: cada actor refería a otro en la «cadena» de la política. Las recipientes se encontraron en su casa, en lugares neutros como la aula del juez de paz de su comunidad o en centros de salud. 


\section{LOS BONOS}

Los bonos - el Bono Renta Dignidad, el Bono Juancito Pinto (BJP) y el Bono Juana Azurduy (BJA) - fueron presentados por el presidente de Bolivia, Evo Morales como los pilares de una política social nueva y universalista. Este artículo se focaliza en el BJP y el BJA porque son transferencias condicionales de efectivo, mientras que el Bono Renta Dignidad es una pensión no contributiva para ancianos después de la edad de 65 años.

Retomando las principales reivindicaciones de los movimientos sociales que los llevaron al poder, las primeras reformas del Movimiento al Socialismo (MAS) y del gobierno de Evo Morales consisten en la «nacionalización»2 de los hidrocarburos y en la nueva Constitución del Estado Plurinacional de Bolivia. En poca palabras, la «nacionalización» permite al Estado romper su dependencia con la ayuda internacional y financiar políticas públicas, entre otros los Bonos (Gray Molina, 2007). Por otro lado, la nueva Constitución del Estado Plurinacional de Bolivia reconoce un nuevo sujeto político: «ndígena, originario y campesinos»3. De esta Constitución surge el Plan Nacional de Desarrollo «Bolivia Digna, Soberana, Productiva y Democrática para Vivir Bien4» (PND) que concibe «una política de protección social de desarrollo integral comunitario» con el objetivo de erradicar la pobreza extrema. Si bien esta nueva política incluye proyectos a largo plazo, tales como «Comunidad en Acción», «Plan Vida» 0 el «Plan de Erradicación de la Extrema Pobreza» (PEEP), solo los Bonos y la Renta Dignidad han sido concretados, en parte debido a los debates teóricos y a las pugnas entre diferentes modelos de desarrollo dentro del Ministerio de Planificación del Desarrollo (CanavireBacarreza \& Ayaviri, 2010: 36).

El Bono Juancito Pinto (BJP) fue creado en 20065. Se enfoca en todos los niños y niñas de los primeros ocho niveles de primaria en la educación pública, contraponiendo el aviso del Banco Mundial que proponía focalizarlo sobre los más pobres6. El BJP tiene por objetivo luchar contra el absentismo escolar y el trabajo infantil. Concede alrededor de 30 \$US al año a los niños, condicionado a su inscripción en la enseñanza pública y a terminar el año escolar con un nivel de asistencia de al menos el 80\% (UE, 2008: 1). Desde 2006, el Bono se

2 Se trata de una renegociación de los contratos con las transnacionales explotadoras de los hidrocarburos más que de una expropiación,

3 El artículo 30 de la Constitución Política del Estado Plurinacional crea una nueva categoría de «nación y pueblo indígena, originario y campesinos» que constituyen «toda la colectividad humana que comparte identidad cultural, idioma, tradición histórica, instituciones, territorialidad y cosmovisión, cuya existencia es anterior a la invasión colonial española». Como lo destaca Lacroix (2011: 7), este artículo unifica «categorías socio-étnicas regionales históricas para superar las diferenciaciones terminológicas (originario o indígenas o campesina) y políticos (pueblos o naciones)». En la continuidad de esta contribución el término «indígena» se refiere a esta categoría.

4 Creado por el Decreto Supremo 29272 del 12 de septiembre de 2007.

5 Decreto Supremo n. 28899 del 26 de octubre de 2006.

6 Entrevista del 30 de agosto de 2010 con la primera responsable del BJP. 
ha extendido a todos los niveles de la escuela pública. Cubre un $17,5 \%$ de la población total; $32,4 \%$ de ella es pobre y $59,7 \%$ indigente (Cecchini \& M adariaga, 2011). El presupuesto anual para 2010 fue de 54,5 millones $\$ U$ S (Weisbrot et al., 2009: 16), lo que representa un 1,16\% del PIB (Marco Navarro, 2013: 27).

El Bono Juana Azurduy (BJA), constituye el más pequeño denominador común de las políticas sociales imaginadas pero no concretadas por el gobierno. De hecho, las diferentes políticas concebidas en el Ministerio de Planificación del Desarrollo para operationalizar el PND incluyeron un Bono enfocado en la relación madreniño. Por ejemplo, con una donación de 30 millones \$USA del Banco Mundial, el PEEP elaboró un proyecto piloto de un bono «madre-niño» focalizado en los 52 municipios más pobres del país con el objetivo de reducir la mortalidad y la desnutrición materna-infantil. El Presidente decidió unilateralmente extenderlo a todo el país y nace el Bono Juana Azurduy?.

El BJA fue creado en mayo de 20098. Madres o mujeres gestantes reciben el equivalente de 260 \$USA en un periodo de 33 meses, los cuales son transferidos durante los controles prenatales, el parto institucional y los controles posnatales. El BJA constituye el $1,41 \%$ de los gastos sociales que representan el $0,22 \%$ del PIB. El BJA se dirige al 3,5\% de la población total, $6 \%$ de los pobres y $10 \%$ de los pobres extremos (Cecchini \& Madariaga, 2011). También emplea alrededor de 600 médicos, dedicados exclusivamente al BJA, a fin de garantizar su aplicación y seguimiento.

Este artículo analiza las representaciones sociales de las beneficiarias y de los responsables políticos de los Bonos al respecto de las relaciones de género y de las condiciones.

\section{CONSENSO MATERNALISTA}

Un elemento consensual entre los responsables políticos y las receptores de los Bonos es apuntar a las mujeres (y no a los hombres) como receptoras de las transferencias. Construido a finales de los años 1990, después de la Conferencia de Beijing, los TCM se declaran «sensible al género» (Molyneux, 2008: 22). Se basaban en el supuesto de que dar dinero a las mujeres les daba más poder. Además, en Bolivia, se concibió la entidad de despatriacalización a dentro del vice-ministerio de descolonización. Sin embargo, esta alusión al poder ganado por las mujeres es retórica y su discurso se encuentra enmarcado en un enfoque maternalista. Se fundamenta en la creencia de larga duración de que la provisión de beneficios a las madres beneficiará a los niños más que las transferencias 0 ingresos que van a padres 0 al hogar en general.

7 Entrevista del 13 de agosto de 2010 con el Coordinador del BJA para el departamento de La Paz.

${ }^{8}$ Creado por el Decreto Supremo 0066 del 3 de abril de 2009. 
Dos concepciones subyacentes de las diferencias de género se utilizaron para justificar este maternalismo. En primer lugar, los responsables de los Bonos asumen la existencia de una clara diferencia entre mujeres y hombres, así como entre padres y madres en relación a los niños. Es visto como rentable pagar la transferencia a las madres para mejorar el bienestar de la infancia. Este enfoque instrumental es muy presente, como lo ilustra la siguiente cita del jefe operativo BJA:

Generalmente en Bolivia y en todo lugar, la que está más relacionada con la salud es la madre que el papá. La madre tiene más relacionamiento con el hijo para llevarlo al establecimiento de salud, no así el papá. Y lo que se ha querido es reforzar eso. Digamos, el poder de la mamá que tiene sobre la salud (13.08.2010, La Paz).

Las receptoras de los Bonos aceptan este papel de «intermedio», precisamente porque también se representan a sí mismas exclusivamente como madres a cargo de sus hijos e hijas. Por ejemplo, una receptora del BJP reitera que debe concederse el dinero a las madres porque lo gasta en el interés de los niños:

Porque nosotras, las mujeres, podemos manejarlo y si lo recibimos, lo gastamos para nuestros hijos, para comprarlos lo que necesitan (09.03.2009, El Alto).

A este argumento de «eficiencia», se añade otro que subraya la segunda concepción de las diferencias de género atribuyendo un valor moral a los sexos. En una representación estricta y dicotómica de los hombres y de las mujeres, los vicios son asociados a los hombres y la virtud a las mujeres. Los hombres son descritos como propensos a gastar los Bonos para fines personales o de fallos, como el alcohol, lo cual justifica excluirlos de los programas de TM C. U na antigua directora del BJA expresa esta desconfianza hacia los hombres con las palabras siguientes:

No lo podemos asegurar al $100 \%$ que el papá no [va utilizar] el dinero y llevárselo para tomar o para celebrar en el famoso viernes de soltero que es tradición en todo el país (26.08.2010, La Paz).

Esta representación está compartida por el coordinador del BJA en La Paz:

El varón la pasa bomba, el viernes aquí en Bolivia, ya tu sabes el viernes de soltero, como hoy día, reciben la plata cobran ya para mi hijito pero festejaremos dos cervecitas $4,8,10,12,6$ de la mañana sigue y ya no hay plata para el bebé [... ] porque la idiosincrasia de nuestro pueblo hace que varón que reciba dinero siempre lo dilapida en licor (13.08.2010, La Paz).

Las mujeres receptoras de los Bonos comparten estas representaciones negativas de los hombres. Por ejemplo, la directora de la organización de las trabajadoras informales de La Paz se refirió también a la tradición de los hombres de celebrar:

Mira, por ejemplo en el Juancito Pinto, debería darse a la madre, ¿no? Porque hay algunos padres que se han pedido el Juancito Pinto el viernes y se han aparecido el martes sin un peso. [... ] Si [el padre] recibe, se va al bar, 6 cervezas y se ha olvidado del Juancito Pinto (09.03.2009, El Alto). 
Cabe destacar que el conjunto de los actores, en todos los niveles de la elaboración e implementación de los Bonos comparten esta representación de los hombres. Lo que surge es una visión abrumadora de los hombres como irresponsables y egoístas, centrados en el ocio y el alcohol. Si bien estas representaciones son negativas para los hombres, más bien refuerzan normas sociales que asignan a las mujeres la responsabilidad de mantener el hogar, que tengan empleo o no.

Mediante la transferencia de los ingresos a las mujeres, los Bonos pueden tener algunos efectos positivos en las relaciones de género (M artínez \& Voorend, 2009). Sin embargo, estos programas dependen en gran medida del trabajo de cuidado no remunerado de las mujeres con fin de mejorar el bienestar de la pequeña infancia. De hecho, una única representación de las relaciones de género domina en el discurso de todos los interlocutores: las mujeres son madres. Los Bonos desalientan a los hombres de asumir el trabajo de cuidado y como este forma parte de los requisitos para beneficiarse de las transferencias, los Bonos sobrecargan las mujeres al mismo tiempo que refuerzan la división sexual del trabajo así como las desigualdades de género (Nagels, 2011).

\section{TENSIONES Y BRECHAS AL RESPECTO A LAS CONDICIONES}

La promoción de la corresponsabilidad se hace especialmente visible por la centralidad de las condiciones, vistas como el reemplazo del «asistencialismo» que caracterizaba los «antiguosprogramas de asistencia social». Estas condicionalidades, centrales en los TMC se orientan en su implementación con miras a modificar el comportamiento de las receptoras. Aquí, ciertos conflictos surgen entre los responsables de los Bonos y las mujeres beneficiarias. Mientras que los primeros estiman que las condiciones de los Bonos deben cambiar las mentalidades y prácticas de las destinatarias, ellas critican las condiciones de este enfoque.

\section{1. El respeto a las condiciones}

En Bolivia, donde las relaciones sociales se estructuran por la intersección de género, raza y clase, las representaciones sociales acerca de las condiciones muestran que la implementación del BJA tiene come objetivo implícito la creación de madres «buenas» y «modernas» que invertirían en sus niños y niñas para prepararlos al mundo contemporáneo. En contradicción con el Estado plurinacional, estas representaciones pertenecen al neocolonialismo que se refiere a la reproducción y a la internalización de las élites latinoamericanas de la matriz cultural e ideológica del Occidente. En esta matriz, la raza es un instrumento de dominación y de diferenciación. Las élites latinoamericanas interiorizan la matriz colonial del poder y del conocimiento: eurocéntrico y «moderno» que niega otros conocimientos propios a los pueblos indígenas (Q uijano, 2000). La operacionalización en el terreno de la perspectiva de inversión social, a través del BJA, conlleva prescripciones normativas sobre el comportamiento maternal 
apropiado que derivan del rechazo neocolonial de las prácticas familiares y reproductivas indígenas, así como de un despido más generalizado de las capacidades de las mujeres indígenas a ser «buenas» madres.

Los discursos de los responsables políticos de los BJP y BJA se basan en la suposición de que las mujeres son ignorantes con respecto a la forma correcta de alimentar y cuidar a sus hijos e hijas y que perpetúan «malas prácticas». De acuerdo con los responsables del programa, las beneficiarias no tienen conocimientos suficientes para practicar una «buena» maternidad. Por ejemplo, un médico a cargo del BJA se queja de la falta de comprensión por parte de las receptoras de cómo ser una buena madre:

A la gente acá se le da las chispitas nutricionales, que son para ayudar a la alimentación: 60 sobres. [... ] Lamentablemente las mamás, a veces, no les dan. Como tienen un sabor no muy agradable entonces el niño no las quiere comer. Lo dejan así y [las madres] dicen, «no, no quiere comer». Y lo dejan así, en la casa, botado. Entonces algunas, todavía, no comprenden la importancia de ese medicamento, de esas chispitas (18.08.2010, La Paz).

En los datos, solo unas cuantas citas se refieren explícitamente a la etnia o a la «ndigeneidad» para explicar la ignorancia o las malas prácticas de las madres, pero el uso despectivo de términos como «comunidad», «idiosincrasia», «mitos» y «creencias» son códigos y referencias a aquello, revelando jerarquías culturales y étnicas. Por ejemplo, los mitos son percibidos como obstáculos para el acceso a los centros de salud, como lo explica un médico implementando el BJA:

Entonces puede ser por eso [que las mujeres no vayan a los centros de salud]: los mitos: que en el médico no te curan, te tratan mal o te hacen más daño (18.08.2010, La Paz).

Del mismo modo, una médica a cargo del BJA utiliza la idiosincrasia para explicar la baja tasa de vacunación entre la población infantil:

Es que tienen esa idiosincrasia de decir de que las vacunas pueden matarles a los niños, que «ustedes están viniendo a matarles a mi hijo, porque tengo varios hijos y tú quieres matármelo a mi hijo». Entonces, bien difícil era llegar a estas señoras (19.08.2010, La Paz).

Frente a lo que ven como ignorancia, los responsables del BJA consideran las condiciones como importantes porque a través de su aplicación pueden modificar las mentalidades y los comportamientos de las destinatarias. Explícitamente, los Bonos sivven para «educar» a la población, como lo expresa un agente del BJA:

Entonces ha sido la parte más difícil: educar a la población. Se les está educando, se les está enseñando (18.08.2010, La Paz).

La educación concierne la higiene, la nutrición y la alimentación, tal cual los explica una iniciadora del BJA en UDAPE9:

Contempla que cuando se hacen los controles de salud del niño, también la madre recibe información sobre, digamos, [... ] prácticas de higiene [... ]

9 Unidad de Análisis de Políticas Sociales y Económicas, centro de investigacion del Ministerio de Planificacion del Desarrollo. 
actitudes saludables, digamos, como hacer el lavado de las manos, como tratar los alimentos (23.08.2010, La Paz).

En concreto, estos médicos se ven como investidos de una misión de enseñar a las madres cómo amamantar y alimentar adecuadamente a sus hijos e hijas:

Además la consejería a la madre de cómo alimentar, cómo amantar, ¿qué es lo que tiene que hacer? [... ]. Se le educa, se le da, se le cita para ver y para ir controlando que vaya mejorando [... se les explica, tardan en entender [... ] se les explica [otra vez] (18.08.2010, La Paz).

El enfoque en la higiene y la educación construye representaciones de estas poblaciones como irresponsables y «sucias». Por otra parte, más allá de la enseñanza de conductas específicas en materia de salud, higiene y nutrición, los Bonos también tratan de promover nuevos estilos de vida entre las receptoras. Como lo comenta una investigadora involucrado en el diseño del BJA, el tema es también sobre el cambio de los hábitos y comportamientos de las pobres:

La idea es de que esto cree hábitos en la población. [... ] Si no que la idea de esto es que se crea un hábito y se educa a la madre. Entonces la madre sabe que llevar a su niño al control le trae un beneficio; más allá de que le den dinero o no (23.08.2010, La Paz).

La misión educativa está tan interiorizada por varios trabajadores locales del BJA que finalmente utilizan las transferencias en efectivo como medios para imponer condiciones extralegales. Por ejemplo, una médica a cargo del BJA intimida a las destinatarias con la amenaza de que sean retirados del programa si sus hijos e hijas no aumentan de peso:

En la que digamos está bajando, entonces le dices, «Señora, si su hijo el siguiente mes no sube [de peso] no entra al bono (19.08.2010, La Paz).

Es un requisito adicional no justificado por ninguna disposición legal para vincular la inclusión en el BJA a la mejora del estado de salud de los niños. Esta propensión por parte de los agentes responsables de la implementación de los Bonos a apoyar e incluso forzar el «desarrollo» de las pobres puede ser explicado por su experiencia personal. Para aquellos con orígenes a menudo modestos e indígenas, ser profesor o médico - en otras palabras, adquirir educación y estatus social superioresexige grandes sacrificios. Entonces, tienden a considerar que las donaciones en efectivo por parte del Estado son inapropiadas. De ahí se incrementan las contrapartes (Rozée, 2007). La lógica desarrollista del gobierno es compartida por los ejecutores locales de los Bonos en contra de cualquier tipo de asistencialismo.

En gran parte, estas representaciones de ignorancia y la necesidad de intervenir en el comportamiento de los hogares surgen del rechazo por parte de los responsables de los Bonos de reconocer el conocimiento derivado de la experiencia y de las prácticas indígenas. Los conflictos sobre las prácticas medicinales, el embarazo y el parto traen estas cuestiones al primer plano. 


\section{2. Biomedicina vs etnomedicina}

Mientras la biomedicina se refiere al conocimiento de Occidente, la medicina andina remite a las cosmovisiones del mundo andino. Cuando la primera se basa en un conjunto de elementos dicotómicos (salud versus enfermedad, cuerpo versus razón, cultura versus naturaleza); la segunda refleja los valores y los sistemas de conocimiento holísticos basados en principios de reciprocidad y de complementariedad (Arnold \& Yapita, 1999). En las prácticas de las poblaciones andinas, ambos sistemas no son ni impermeables ni excluyentes. Los pacientes incorporan elementos de ambos conjuntos y dentro de procesos de negociación flexibles y creativos (Miles \& Leatherman, 2003). Desde 1986, Bolivia fue uno de los pocos países del mundo en legalizar la medicina tradicional. Además, en el Plan Nacional de Desarrollo (2006), un capítulo se dedica a la descolonización de la salud (Ministerio de Planificación del Desarrollo, 2006: 37-43) y los artículos 35, 37 y 39 de la nueva Constitución Política del Estado Plurinacional de Bolivia (2009) mantienen los mismos principios. Sin embargo, el papel de la etnomedicina es poco respetado y reconocido en las prácticas medicinales (Rozée, 2007: 5). La política de salud promueve la biomedicina y tiende a imponerla sobre la etnomedicina. Por ejemplo, mientras que los médicos deben ser capaces de hablar un idioma nativo, un médico del BJA explica que la formación adentro del BJA es limitada:

No todos hablamos aimara [... ] Nos han brindado algunas charlas, algunos cursos [sobre interculturalidad]. No una capacitación profunda que se necesitaría por un tiempo, como por unos meses, por ejemplo. No, es una charla para una semana (18.08.2010, La Paz).

Profesionales de la salud no rechazan la medicina andina en sí pero lo hacen promoviendo exclusivamente la biomedicina institucional. Si se aceptan prácticas de la etnomedicina en los hogares, son vistas como complementarias a la biomedicina que es la medicina privilegiada en los centros de salud, como lo explica un médico BJA:

Claro, porque las señoras, las mamás tienen un punto de vista, a veces: tratan algunas patologías con diferentes métodos caseros. La mayoría no son tampoco malos, no son dañinos, pero deberían ser más bien complementarios en la atención médica (20.08.2010, El Alto).

Estas tensiones entre la biomedicina y la etnomedicina se reflejen en el parto. En pocas palabras, el parto tradicional se refiere a los rituales y las manipulaciones que corresponden a representaciones holísticas e integrales del cuerpo, de la salud y de las personas propias a la cosmovisión andina. Durante el nacimiento, las mujeres están relacionadas con la Pachamama, la «madre tierra». Entonces, tienen que «empujar hacia abajo», por lo tanto con una posición vertical. La partera, el futuro padre y otras personas de confianza acompañan a la parturienta, de ahí la calificación de parto «colectivo». El parto es considerado como un importante rito de pasaje en la vida de las mujeres y de los recién nacidos. El parto institucional va en contra del parto tradicional. Sigue los criterios de la biomedicina y es llevado 
por las normas culturales occidentales en mesas ginecológicas horizontales sin la presencia de la familia o de parientes. La medicalización del parto le da el primer lugar al terapeuta que «roba» el papel protagonista de las mujeres y su poder de dar a luz. Por lo tanto, se reproducen dominaciones étnicas, de clase y de género, entre el médico y la parturienta (La Riva González, 2000).

Según las normas legales de salud intercultural, el parto tradicional debería ser posible en Bolivia. Sin embargo, en la realidad, el parto vertical no es practicado en los centros de salud donde se realizó el trabajo de campo. Un médico a cargo del BJA reconoce que el parto vertical no es permitido en el centro de salud a donde él implementa el BJA:

Eso todavía en el centro no se está acudiendo a cumplir. Como te digo, para empezar la infraestructura que tenemos no es la adecuada (20.08.2010, El Alto).

Otras investigaciones muestran que el personal de salud considera el parto tradicional como peligroso e inadecuado. Agacharse es percibido como una causa de daño al bebe y las aulas de nacimiento son consideradas como demasiadas pequeñas para albergar a la familia (Rozée, 2007; Platt, 2006). Las tensiones sobre el parto reflejan el debate entre la medicina andina y la biomedicina: el personal de salud acepta incorporar elementos de la medicina tradicional, solo cuando no implican cambios en sus prácticas o representaciones de la biomedicina, considerada como la única ciencia médica válida. De hecho, en las universidades la biomedicina es hegemónica; mientras la etnomedicina es ausente, marginalizada y depreciada (U riburu, 2006: 176). Por lo tanto,

una proporción significativa de los médicos no son preparados para asistir a los partos en posiciones tradicionales, por la falta de formación y por una actitud autoritaria de no aceptación de las expectativas y tradiciones culturales (Rozée, 2007: 7).

La salud intercultural defendida por las normas legales se mantiene en el «limbo» de la palabrería oficial. Los implementadores del BJA siguen considerando que las prácticas y la cultura indígenas son responsables de los malos indicadores de salud y la cultura sigue siendo una barrera a la biomedicina, es decir, la «moderna» y el único camino a la «buena» salud. La cultura indígena permanece invocada como una fatalidad, responsable de todos los males, mientras que se desconoce (Rozée, 2007: 7-8). La instrumentalización de la cultura para explicar los problemas relacionados con el acceso a los servicios de salud «transforma en términos culturales prejuicios con fuertes connotaciones racistas» (Fassin, 2001: 9). La atención se focaliza únicamente en los receptores del BJA mientras que la cultura de los actores institucionales está ausente. Por lo tanto, se niegan las relaciones de dominación entre estos dos grupos. Por consiguiente, las relaciones de poder se refuerzan entre, por un lado, los responsables políticos, los expertos y los profesionales del BJA - sobre todo hombres considerados e auto-identificados como «blancos», «mestizos» y de clase media- y por otro lado, las mujeres consideradas e auto-identificadas como «pobres»e «indígenas». 


\section{3. La reacción de las beneficiarias: entre la internalización y la resistencia}

Frente a estas normas y prácticas de los Bonos, las beneficiarias oscilan entre la internalización y la resistencia a estas reglas. La internalización se centra en los objetivos del programa: mejorar el bienestar de los niños y de las niñas10, como una de ellas lo expresa:

¡Uy, sí! Harto ha cambiado para nosotros. Es una alegría, porque tenemos un apoyo a nuestros niños con el bono Juancito Pinto, porque en el campo, hay veces, para ni un lápiz hay dinero. Solo para alimentación tenemos. Eso nos ha apoyado. [... ] harto nos ayuda esto. Este bono Juan Azurduy, también, un incentivo para que haya más atención en los centros de salud. Porque antes no iban a los centros de salud. [... ] Ahora, con ese incentivo, ya van a hacerse atender el parto, a hacerse controlar. Harto nos ayuda (02.09.2010, El Alto).

Estos comentarios positivos solo se refieren a la transferencia de dinero. Las receptoras de los Bonos son muy críticas hacia las condiciones y de ahí construyen resistencias en contra de los roles maternos prescritos. En primer lugar, denuncian los problemas de suministro de servicios de salud: su cantidad insuficiente y su dispersión geográfica. La distancia entre los lugares de residencia y las instituciones relacionadas con el BJA es un problema para las mujeres asignadas a otras tareas productivas, como lo explica una beneficiara del BJP:

Pero siempre hay dificultades. Si podría ser que se pueda crear los bancos en las provincias. Sería mejor, ¿no? [... ] Con eso, cualquier rato [cuando] la mamá tiene tiempo, viene y cobra. Sí, porque el ejército va para dos días. Entonces, hay veces para día de semana no tienen tiempo (02.09.2010, El Alto).

Por otra parte, las receptoras critican la cantidad de dinero recibido, que es insuficiente para alimentar a sus hijos e hijas:

La plata no alcanza porque con 125 bs podemos comprar solo dos cajas de leche (20.08.2010, El Alto).

O tra destinataria del BJA comparte esta opinión, estimando que no tiene sentido asistir a talleres y aprender cómo mejorar la dieta de los niños, ya que ella es financieramente incapaz de obtener más alimentos ricos en proteínas o en vitaminas:

El problema es que damos lo que tenemos en casa. No veo por qué ir a estos talleres (para luchar contra la desnutrición) ya que no puedo comprar más frutas, verduras o pollo (20.08.2010, El Alto).

10 Está vinculado a la internalización y la reproducción por las receptoras del estereotipo marianista que les hacen verse primero como madres responsables de sus niños y niñas (Nagels, 2011). 
Aquellas críticas son estructurales: los programas no toman en cuenta las condiciones materiales en las cuales las receptoras viven. Por otra parte, las receptoras consideran que el BJA no cumple con sus propias obligaciones en términos de planificación familiar y de parto vertical11.

El acceso a la modernidad, a través del acceso a la maternidad «occidental» donde la realización personal tiene prioridad no puede concretarse frente a la falta de métodos anticonceptivos. Mientras el BJA afirma difundir información sobre anticonceptivos, no lo es, tal lo expresa una receptora del BJA:

He calculado. Me dijeron de venir después del nacimiento y el regreso de mi periodo para hacer preguntas (20.08.2010, El Alto).

Antes de la consulta, expresó su deseo de preguntar al médico cómo podía planificar sus embarazos, pero durante la consulta, ella no preguntó. Explicó su actitud de la siguiente manera:

El problema es que los médicos no tienen tiempo para responder a nuestras preguntas (20.08.2010, El Alto).

Parece que la sumisión y la timidez de las mujeres hacia los trabajadores de salud responden a lo que piensan que los médicos esperan de ellas. En otras palabras, ellas se conformen a la imagen de las mujeres indígenas como «ignorantes». Sin embargo, según Dibbits (2003: 151), la sumisión a los funcionarios públicos puede ser funcional en términos de autoprotección sin aceptar su inferioridad social. «No hablar ni preguntar» evita más intrusión de los médicos en su vida privada. Es interesante notar que estas mujeres juzgadas como premodernas, comparten reivindicaciones generalmente asociadas a las feministas «mestizas» y «modernas»: el control de la natalidad. Siguiendo con el tema de los derechos sexuales y reproductivos, no se permite el parto vertical o de cuclillas, según lo explicado por una receptora del BJA:

Di a luz aquí, y no respetan el parto tradicional, pero mi marido era capaz de entrar en la habitación. El parto es mejor en casa porque nos cuida durante 7 días (20.08.2010, El Alto).

Reivindicando información sobre la planificación familiar o para poder dar a luz «como en casa», las receptoras aprovechan los compromisos del BJA para denunciarlo. Ellas se apropian requisitos institucionales (la anticoncepción y el parto institucional respetuoso) para construir márgenes y denunciar que son víctimas de abusos (Faya Robles, 2008).

Más allá de los problemas institucionales y estructurales, las receptoras denuncian los abusos y el mal trato que viven en los centros de salud. Los comportamientos de los trabajadores de salud faltan de respeto, son discriminatorios, sexistas, y abusivo. No trabajan, las hacen esperar, ríen y no las respetan. La imposibilidad de acceder a los centros de salud es especialmente problemática cuando los centros

11 El BJA debe proporcionar información de planificación familiar y el parto vertical. 
de salud se ubican a grandes distancias de los hogares receptoras, como lo ilustran varias citas de las receptoras del BJA.

El personal de salud tiene bastante poder sobre lasmujeres receptoras. La movilidad social, representada por un título, seguido por un trabajo remunerado, más aún en el campo de la medicina, es valorada socialmente. En contextos donde el género, las condiciones sociales y étnicas se entrelazan, el mayor estatus social significa denigrar una parte de su «indigeneidad» (Q uijano, 1980; Rousseau, 2009).

Las críticas y resistencias se hacen explícitas cuando las mujeres desarrollan estrategias de derivación con respeto a las condiciones de los Bonos sin dejar de ser beneficiarias de esta política social. U na resistencia explícita se refiere por ejemplo al pluralismo médico combinando biomédica y etnomedicina. El uso del pluralismo médico por parte de la receptora permite resistir a la imposición de la biomedicina prescrito por el programa.

Por lo tanto, ir a los centros de salud y cumplir con las condiciones del programa se enmarcan en un pragmatismo no exclusivo. Esto se confirma por dos receptoras de BJA. Una reconoce mientras risitas, que si no fuera por el atractivo financiero, ella no iría a los centros de salud:

[Ja, ja], sí de verdad, yo sin bono no vendría.

O tra receptora, es al mismo tiempo en favor de la mejora del bienestar de su hija y no parece demasiado preocupada de que su hija está en un estado de desnutrición:

No, no me importa, porque mis otros hijos también eran demasiado pequeños pero recuperan [... ]. Mi bebé es demasiada pequeña y no muy grande, pero ella está en el proceso de recuperación.

Por lo tanto, las mujeres son muy conscientes de lo que tienen que decir para permanecer en el programa (por ejemplo al respecto de la mejora de la nutrición infantil) mientras continúa su propia estrategia fundamental: sobrevivir.

Por último, la mayor resistencia a los Bonos es la opción de salida, como lo explica un representante vecinal comunitario en El Alto:

Eso de Juana Azurduy como lo digo uno va y otros no van no le dan importancia (...) no quieren ir llevar nomas [...] se aburren estar ahí, haciendo cola, a algunas les cuesta estar paradas y se aburren. Así que no vale los $50 \mathrm{Bs}^{12}$. [... ] en esa forma un poquito la gente así ya no quiere no, para que hacer cola, y no más van.

Si el retiro voluntario del programa consiste en la estrategia de rechazo de las condiciones que implican cambios sustanciales en la vida de las mujeres pobres, no es posible para las más humildes de las receptoras. Estas últimas no tienen otra opción que aceptar el cumplimiento de las condiciones y todos los maltratos y humillaciones que acompañan su aplicación. 


\section{DESARRO LLISMO, NEO CO LO NIALISMO Y ETNO CENTRISMO}

Los responsables políticos y los ejecutores utilizan los Bonos como instrumentos coercitivos con el objetivo de controlar las poblaciones indígenas con fines higienista y de asimilación, reproduciendo entonces el neocolonialismo. La continuación del neocolonialismo en la era de la «descolonización del Estado», reclamada por la administración de Evo Morales parece contradictoria. Una explicación de esta contradicción se encuentra en la supremacía de las lógicas desarrollistas dentro del gobierno evacuando los referentes a la perspectiva del «buen vivir» presente al inicio de la presidencia de Morales y en la Constitución del nuevo Estado Plurinacional de Bolivia.

La lógica desarrollista13 promueve la rehabilitación de un Estado fuerte como agente de desarrollo a través de mega proyectos petroquímicos, mineros, carreteros e hidroeléctricos (Lavaud, 2009). Este modelo pone una fuerte énfasis en la explotación de los recursos naturales y las políticas productivistas que van en contra de la preservación del medio ambiente propio del «buen vivir». Cualesquiera sean los matices, este modelo desarrollista privilegia la explotación de los recursos naturales y las políticas de productividad. En contraste, la perspectiva del «buen vivir» se estructura en discursos socioambientalistas basados en el pluralismo étnico social, jurídico, económico y político. Cualesquiera sean sus matices, esta perspectiva se opone a proyectos productivistas y a la explotación de los recursos naturales (Stefanoni, 2011). Los acontecimientos del TIPNIS (Territorio Indígena y Parque Isidoro Sécure) en 2011 muestran que la balanza se inclinó más por el lado del «desarrollismo» que del «buen vivir»14. El desarrollismo reproduce el neocolonialismo y la búsqueda de la modernidad solo entendida como un concepto occidental.

Sin embargo, las receptoras se niegan a someterse por completo a este neocolonialismo expresado por las condiciones que se les imponen desde fuera, pero aceptan una parte de ellas a fin de mejorar su vida cotidiana. Según Lautier (2009: 33), aceptan e incluso reivindican la ayuda pero «vomitan la moral y los estilos de vida que se tratan de vender con la ayuda». A través de estos procesos, las mujeres cuestionan las representaciones de los responsables de los Bonos que los construyen como «ignorantes», «pasivas» y con «prácticas pre-modernas». Así que ellas denuncian el neocolonialismo y las dominaciones étnicas y de género

${ }^{13}$ El desarrollismo viene de la teoría económica de la CEPAL después de la Segunda Guerra M undial y la promoción en América Latina del modelo de desarrollo ISI de industrialización para la sustitución a las importaciones.

${ }^{14}$ En septiembre de 2011, el gobierno hizo uso de la fuerza en contra de movilizaciones populares indígenas opuestas a la construcción de una carretera a través del territorio indígena de este parque. Estas posiciones gubernamentales apoyan las declaraciones de julio de 2011 del Vicepresidente que estigmatizaban los portavoces del vivir bien como actores sumisos a ONG ambientalistas occidentales. En otro momento el Vicepresidente declaró sin matices que el derecho del Estado es superior a los derechos territoriales de los pueblos amazónicos con respecto a la explotación de los recursos naturales (Lavaud, 2009: 101). 
se entrelazan y se refuerzan mutuamente. Juegan en los márgenes para crear intersticios de poder en los cuales pueden existir como mujeres indígenas, en proceso de cambio y de renegociación constante. De este modo, pueden mejorar un poco sus condiciones de vida, a través del acceso a los Bonos, mientras que se oponen a las dominaciones sociales, étnicas y de género que viven haciendo parte de los Bonos.

Tres observaciones concluirán este capítulo. Aunque en el corto plazo, estos programas podrían mejorar los indicadores de pobreza - como afirman las instituciones internacionales de desarrollo- los TM C no luchan contra sus causas estructurales, reproduciendo el neocolonialismo sexista. En la misma línea que la obra de Gautier \& Q uesnel (1993) en México u O livier de Sardan (2013) en Níger, destacando las diferencias entre las políticas de desarrollo y su traducción práctica, estas observaciones resaltan el carácter coercitivo de esta política y la reproducción de las desigualdades étnicas, de clase y de género.

En primer lugar, transferir el dinero a las mujeres, expulsando a los hombres de todas las tareas reproductivas y de cuidado, refuerza las desigualdades de género. Sobrecargada por el respeto de los requisitos, las mujeres no tienen tiempo para estudiar o trabajar. La invisibilidad ante las necesidades de las mujeres puede ser contraproducente para el bienestar de los niños. La investigación muestra que el acceso de las mujeres a la educación, la salud y el mercado laboral mejora las condiciones de vida de sus hijos. Sin embargo, esta dimensión de lucha contra la pobreza es ignorada por los TCM.

En segundo lugar, las desigualdades sociales se reproducen en la medida en que la pobreza estructural no se aborda. El silencio sobre la pobreza de las destinatarias finales es sorprendente en la medida que son focalizadas por los Bonos porque son pobres. La pobreza sigue siendo explicada de forma individual y las pobres son las responsables de su situación. Todos los obstáculos relacionados con la prestación del servicio son invisibles en el diseño y la implementación de los Bonos.

El neocolonialismo reproduce las desigualdades étnicas. La medicina intercultural permanece ausente en las prácticas médicas. La interculturalidad implica una relación de real interacción dialógica entre diferentes actores, titulares de diferentes universos simbólicos. Puede generar algo nuevo que enriquece a ambas partes sin pérdida de uno y otro lado. Ambos grupos expanden sus horizontes simplemente aceptando al otro. La interculturalidad no concibe la cosmovisión andina como una barrera, sino como un conocimiento en las mismas condiciones que la ciencia occidental (Albó, 2004: 66).

Las receptoras, sin negar la discriminación étnica, insisten en sus duras condiciones de vida que los Bonos no toman en cuenta. Como Ramírez (2009: 144) lo enfatiza, si lo «cultural» es fundamental, «el elemento central que aparece es la violencia estructural del sistema de salud». De hecho, como se ha visto, cuando la mujer indígena quiere ir a un servicio de salud, ella se enfrenta a varios obstáculos: el transporte, el costo, los maltratos, etc. Estos parecen mucho más materiales o sociales que culturales. U na vez allí, las condiciones en las que son recibidas son insuficientes. Los centros de salud son lugares privilegiados donde se expresa la 
violencia del colonialismo y del sexismo. Los trabajadores de salud y los médicos, la mayoría de ellos identificados como «blancos» o «mestizos», reproducen desigualdades sociales, étnicas y de género en contra de las mujeres indígenas.

\section{CONCLUSIÓN}

El enfoque de la socio-antropología del desarrollo permite destacar y comprender características a primera vista paradójicas de las políticas sociales implementadas por el primer gobierno «indígena» de Bolivia. De hecho, analizar las políticas sociales y de desarrollo como objeto de investigación mediante el estudio de las interacciones entre desarrolladores y desarrollados permite aclarar las brechas de implementación entre las normas oficiales del Estado plurinacional y las normas prácticas. Mientras el Estado plurinacional, desde la nueva Constitución hacia las normas ministeriales, promueve las descolonización y la despatriacalización del Estado y de la sociedad, sus acciones, mediante sus políticas públicas, conllevan representaciones que reproducen tanto el maternalismo que el neocolonialismo. Estas brechas de implementación se pueden explicar no solo por las tensiones dentro del gobierno de Morales sino también por el hecho de que el desarrollo y sus políticas, siguiendo el enfoque de socio-antropología del desarrollo, son tanto estrategias como recursos por sus actores. Tantos los médicos como las receptoras de los Bonos los utilizan de acuerdo a sus intereses y representación de lo que es el desarrollo.

\section{Referencias citadas}

ALBÓ , X., 2004 - Interculturidad y salud. In: Salud e interculturalidad en América Latina. Perspectivas antropológicas (G. Fernández Júarez, ed.): 65-74; Quito: Abya-Yala, Universitad de Castilla de la Mancha.

ARNOLD, D. \& DIOS YAPITA, J., 1999 - Vocabulario aymara del parto y de la vida reproductiva de la mujer, 286 pp.; La Paz: ILCA, Family Health International.

BARRIENTOS, A., 2009 - Labour markets and the (hyphenated) welfare regime in Latin America. Economy and Society, 38 (1): 81-108.

BARRIENTOS, A. \& DEJONG, J., 2006 - Reducing Child Poverty with Cash Transfers: A Sure Thing? Development Policy Review, 24 (5): 537-552.

BASTAGLI, F., 2009 - From Social Safety Net to Social Policy? The Role of Conditional Cash Transfers in Welfare State Development in Latin America; Brasilia: International Policy Center for Inclusive Growth. UNDP.

CAN AVIRE-BACARREZA, G. \& AYAVIRI, M. M., 2010 - Políticas macroeconómicas, choques externos y protección social en Bolivia; La Paz, UDAPE.

CECCHINI, S. \& MADARIAGA, A., 2011 - Programas de transferencias condicionadas. Balance de la experiencia reciente en América Latina y el Caribe, 110 pp.; Santiago de Chile: CEPAL, ASDI. 
DIBBITS, I., 2003 - Uno de los dos: el involucramiento de los hombres en la atención de la salud perinatal, revelaciones desde Santa Rosa Pampa, el Alto, Bolivia, 235 pp.; La Paz: Tahimapu.

FASSIN, D., 2001 - Le culturalisme pratique de la santé publique. Critique d'un sens commun. In: Critique de la santé publique. Une approche anthropologique (D. Fassin \& J.-P. Dozon, eds.): 181-208; París: Balland.

FAYA RO BLES, A., 2008 - L'«humanisation de l'accouchement et de la naissance au Brésil»: de nouveaux dispositifs de régulation des femmes pauvres ? Lien social et politiques. Corps et politiques : entre l'individuel et le collectif, 59: 115-124.

FISZBEIN, A., SCHADY, N., FERREIRA, F., GROSH, M., KELEHER, N., OLINTRO, P. \& SKO UFIAS, E., 2009 - Conditional Cash Transfers. Reducing Present and Future Poverty, xviii + 362 pp.; Washington, D.C.: World Bank.

GAUTIER, A. \& Q UESNEL, A., 1993 - Politique de population, médiateurs institutionnels et régulation de la fécondité au Yucatan (Mexique), 113 pp.; París: Institut francais de recherche scientifique pour le développement en coopération, El colegio de México.

GRAY M O LINA, G., 2007 - El reto posneoliberal de Bolivia. Nueva Sociedad, 209 (mayojunio): 118-129.

JENSO N, J., 2010 - Diffusing Ideas for After Neoliberalism: The Social Investment Perspective in Europe and Latin America. Global Social Policy, 10 (1): 59-84.

JENSO N, J. \& SAINT-M ARTIN, D., 2006 - Building blocks for a new social architecture: the LEGOTM paradigm of an active society. Policy \& Politics, 34 (3): 429-451.

JO DELET, D., 1989 - Les représentations sociales, 447 pp.; París: P.U.F.

LACROIX, L., 2011 - État plurinational et redéfinition du multiculturalisme en Bolivie. In: Le multiculturalisme au concret. Un modèle latino-américain ? (C. Gros \& D. Dumoulin Kervan, eds.): 135-146; París: Presses de la Sorbonne N ouvelle.

LA RIVA GONZÁLEZ, P., 2000 - Le Walthana Hampi ou la reconstruction du corps, conception de la grossesse dans les Andes du Sud du Pérou. Journal de la Société des Américanistes, 86: 169-184.

LAUTIER, B., 2009 - Le consensus sur les politiques sociales en Amérique latine, négation de la démocratie ? In: Congrès AFSP. Les politiques sociales: mutations, enjeux, théories: 1-13.

LAVAU D, J.-P., 2009 - Indianisme et écologie dans les pays andins: dispositif légal, discours officiels et mobilisations. Problèmes d'Amérique latine, 76: 97-117.

LO PREITE, D. \& MACDO NALD, L., 2014 - Gender and Latin American Welfare Regimes: Early Childhood Education and Care Policies in Argentina and Mexico. Social Politics, 21: 1-23.

MARCO NAVARRO, F., 2013 - El Bono Juancito Pinto del Estado Plurinacional de Bolivia. Programas de transferencias monetarias e infancia; Santiago de Chile: CEPAL.

MARTINEZ FRANZONI, J., 2008 - Welfare regimes in Latin America: capturing constellations of markets, families and policies. Latin American Politics and Society, 50 (2): 67-100.

MARTINEZ FRANZO NI, J. \& VO O REND, K., 2009 - The Role of Distributional Coalitions in Welfare Regimes: Chile, Costa Rica and El Salvador. Social Policy and Administration, 43 (4): 364-381.

MILES, A. \& LEATHERM AN, T., 2003 - Perspectives on medical anthropology in the Andes. In: Medical Pluralism in the Andes. Theory and Practices in Medical Anthropology and International Health (J. D. Koss-Chioino, T. Leatherman \& C. Greenway, eds.): 3-15; London, New-York: Routledge. 
MINISTERIO DE PLANIFICACIÓ N DEL DESARRO LLO, 2006 - Plan Nacional de Desarrollo. Bolivia digna, soberana, productiva y democrática para Vivir Bien; La Paz: Ministerio de Planificación del Desarrollo.

M O LYNEUX, M ., 2008 - Conditional Cash Transfers: A Pathway to Women's Empowerment? Pathways of Women's Empowerment, 5: 1-92.

NAGELS, N., 2011 - Les représentations des rapports sociaux de sexe au sein des politiques de lutte contre la pauvreté au Pérou. Recherches féministes, 24 (2): 115-134.

NAGELS, N., 2013 - Genre et politiques de lutte contre la pauvreté au Pérou et en Bolivie: quels enjeux de citoyenneté ?; Ginebra: Graduate Institute. PhD.

NAGELS, N., 2014 - Programmes de transferts conditionnés au Pérou et en Bolivie : entre ciblage et universalisation de l'assistance sociale. Revue internationale de politique comparée, 21 (1): 111-132.

OIT, 2011 - Social Protection Floor for Fair and Inclusive Globalization. Report of the Advisory Group chaired by Michelle Bachelet Convened by the ILO with the collaboration of the WHO; Geneva: Organisation Internationale du Travail (Bachelet Report).

O LIVIER DE SARD AN, J-P., 2001 - Les trois approches en anthropologie du développement. Tiers-M onde, 42 (168): 729-754.

OLIVIER DE SARDAN, J.-P., 2013 - Les transferts monétaires au Niger : la manne et les soupcons. Synthèse des recherches menées par le LASD EL, 70 pp.; Niamey: LASDEL. Études et travaux, 108.

PLATT, T., 2006 - El feto agresivo. Parto, formación de la persona y mito-historia en los Andes. In: Salud e interculturidad en América Latina. Antropología de la salud y crítica intercultural (G. Fernández Juárez, ed.): 145-172; Q uito: Abya-Yala, U niversitad de Castilla de la Mancha.

QUIJANO, A., 1980 - Dominación y cultura. Los cholos y el conflicto cultural en el Perú, 119 pp.; Lima: Mosca Azul Editores.

QUIJANO, A., 2000 - Colonialidad del poder, eurocentrismo y América Latina. In: La colonialidad del saber: eurocentrismo y ciencias sociales. Perspectivas latinoamericanas (E. Lander, ed.): 201-246; Buenos Aires: CLASCO.

RAM ÍREZ HITA, S., 2009 - Calidad de atención en salud. Prácticas y representaciones sociales en la población quechua y aymará del altiplano boliviano; La Paz: OPS, OMS.

RAZAVI, S., 2014 - Addressing/reforming care, but on whose terms? In: N ew Frontiers in Feminist Political Economy (R. Shirin \& G. Waylen, eds.); Milton Park: Routledge.

ROUSSEAU, S., 2009 - Genre et ethnicité racialisée en Bolivie : pour une étude intersectionnelle des mouvements sociaux. Sociologie et sociétés, 41 (2): 135-160.

RO ZÉE, V., 2007 - Les patrons culturels du comportement reproductif et sexuel dans les Andes Boliviennes. Cuestiones del tiempo presente, Número 7: 1-13.

SKO U FIAS, E. \& MC CLAFFERTY, B., 2001 - Is PRO GRESA Working? Summary of the Results of an Evaluation, IFPRI. International Food Policy Research Institute.

STAAB, S., 2010 - Social Investment Perspective in Chile and Latin America: Towards Equal O pportunities for Women and Children? Journal of Social Policy, 39 (4): 607-626.

STEFAN O NI, P., 2011 - Les antinomies du «bien vivre». France Amérique latine magazine, 105: 17-18.

SU GIYAMA, N., 2011 - The diffusion of Conditional Cash Transfer programs in the Americas. Global Social Policy, 11 (2-3): 250-278. 
Género y apropiación de la perspectiva de inversión social en Bolivia: el sistema de los Bonos

THE ECO NOMIST, 2010 - How to get children out of jobs and into school. The limits of Brazil's much admired and emulated anti-poverty programme. The Economist; São Paulo: The Economist.

URIBURU, G., 2006 - Mortalidad materna en Bolivia. ¿Qué hacer para evitar tantas muertes de mujeres? In: Salud e interculturidad en América Latina. Antropología de la salud y crítica intercultural (G. Fernández Juárez): 173-227; Quito: Abya-Yala, Universitad de Castilla de la Mancha.

UNIDAD EJECUTIVA DEL BO NO JUANCITO PINTO, 2008 - Informe de cierre preliminar del Bono Juancito Pinto al 31 de diciembre 2008; La Paz: M inisterio de Educación y de Cultura.

VILLATORO S., P., 2004 - Programas de reducción de la pobreza en América Latina. Un análisis de cinco experiencias. Políticas sociales, CEPAL, 87: 1-40; Santiago de Chile: CEPAL.

WEISBROT, M ., RAY, R. \& JOHNSTO N, J., 2009 - Bolivia: The Economy During the Morales Administration, 33 pp.; Washington D.C: Center for Economic and Policy Research. 